\title{
BMJ Open Measuring outcome differences associated with STEMI screening and diagnostic performance: a multicentred retrospective cohort study protocol
}

\author{
Maame Yaa A B Yiadom, ${ }^{1}$ Bryn E Mumma, ${ }^{2}$ Christopher W Baugh, ${ }^{3}$ \\ Brian W Patterson, ${ }^{4}$ Angela M Mills, ${ }^{5}$ Gilberto Salazar, ${ }^{6}$ Mary Tanski, ${ }^{7}$ \\ Cathy A Jenkins, ${ }^{8}$ Timothy J Vogus, ${ }^{9}$ Karen F Miller, ${ }^{1}$ Brittney E Jackson, ${ }^{1}$ \\ Christoph U Lehmann, ${ }^{10}$ Stephen C Dorner, ${ }^{3}$ Jennifer L West, ${ }^{1}$ Thomas J Wang, ${ }^{11}$ \\ Sean P Collins, ${ }^{1}$ Robert S Dittus, ${ }^{12}$ Gordon R Bernard, ${ }^{13}$ Alan B Storrow, ${ }^{1}$ \\ Dandan $\mathrm{Liu}^{8}$
}

To cite: Yiadom MYAB, Mumma BE, Baugh CW, et al. Measuring outcome differences associated with STEMI screening and diagnostic performance: a multicentred retrospective cohort study protocol. BMJ Open 2018;8:e022453. doi:10.1136/ bmjopen-2018-022453

- Prepublication history for this paper is available online. To view these files, please visit the journal online (http://dx.doi. org/10.1136/bmjopen-2017022453).

Received 1 March 2018 Revised 6 March 2018 Accepted 8 March 2018

Check for updates

For numbered affiliations see end of article.

Correspondence to Dr Maame Yaa A B Yiadom; maya.yiadom@vanderbilt.edu

\section{ABSTRACT}

Introduction Advances in ST-segment elevation myocardial infarction (STEMI) management have involved improving the clinical processes connecting patients with timely emergency cardiovascular care. Screening upon emergency department (ED) arrival for an early ECG to diagnose STEMI, however, is not optimal for all patients. In addition, the degree to which timely screening and diagnosis are associated with improved time to intervention and postpercutaneous coronary intervention outcomes, under more contemporary practice conditions, is not known.

Methods We present the methods for a retrospective multicentre cohort study anticipated to include 1220 patients across seven EDs to (1) evaluate the relationship between timely screening and diagnosis with treatment and postintervention clinical outcomes; (2) introduce novel measures for cross-facility performance comparisons of screening and diagnostic care team performance including: door-to-screening, door-to-diagnosis and doorto-catheterisation laboratory arrival times and (3) describe the use of electronic health record data in tandem with an existing disease registry.

Ethics and dissemination The completion of this study will provide critical feedback on the quality of screening and diagnostic performance within the contemporary STEMI care pathway that can be used to (1) improve emergency care delivery for patients with STEMI presenting to the ED, (2) present novel metrics for the comparison of screening and diagnostic care and (3) inform the development of screening and diagnostic support tools that could be translated to other care environments. We will disseminate our results via publication and quality performance data sharing with each site. Institutional ethics review approval was received prior to study initiation.

\section{INTRODUCTION}

We can find opportunities to improve ST-segment elevation myocardial infarction

\section{Strengths and limitations of this study}

- This study overcomes the lack of adequate data within existing national registries to study STsegment elevation myocardial infarction (STEMI) screening and diagnosis.

- It presents a structured approach to multicentred retrospective data collection for a low frequency, but critical, emergency condition.

- Despite studying STEMI care in seven tertiary care academic facilities, study result will inform the STEMI screening and diagnostic practices of more diverse emergency departments as well as other environments with patients reporting acute symptoms suggestive of STEMI.

(STEMI) care by exploring the timeliness of screening and diagnosis. Each year, approximately 258000 patients present to an United States emergency departments (ED) with STEMI. ${ }^{1}$ Advances in STEMI care have involved improvements in the clinical processes connecting patients-experiencing this rapidly progressive pathophysiologywith timely emergency care. ${ }^{2-10}$ The completion of screening upon ED arrival for an early ECG to diagnose STEMI, however, is not optimal for all patients. ${ }^{11-14}$ This is particularly the case when studying the percutaneous coronary intervention (PCI) centre affiliated ED subpopulation, where variation attributed to interfacility transfer is removed. Despite proximity to the location of intervention, timely care is highly dependent on the pre-existing screening, diagnosis and treatment systems. ${ }^{61114}$ Here, we present the methods for our multicentre investigation to (1) evaluate the relationship between timely 
screening and diagnosis with treatment time and clinical outcomes, (2) characterise generalisable screening and diagnostic measures that can be used for cross-facility performance comparisons and (3) describe the use of electronic health record (EHR) data in tandem with an existing disease registry.

Given the European Heart Association, American College of Cardiology and American Heart Association recommendation to obtain an early ECG within 10 min for patients with symptoms suggestive of STEMI, ${ }^{2-4}$ nearly $85 \%$ of EDs have protocols to guide the screen of all arriving patients for the need of an early ECG. ${ }^{14}$ We define an early ECG as one performed upon ED arrival, typically well before physician evaluation, to diagnose STEMI in a timely fashion. ${ }^{14-16}$ Our prior work identified $12.8 \%$ (95\% CI $3.4 \%$ to $32.6 \%$ ) of patients with STEMI do not receive a timely ECG. The resulting diagnostic delay led to 14-80 minutes of additional myocardial ischaemia time. ${ }^{14}$ Earlier treatment has been historically associated with better outcomes. ${ }^{23}$ The degree to which timely screening and diagnosis are associated with improved time to intervention and post-PCI outcomes, under more contemporary practice conditions, is not known. In addition, STEMI care pathway performance has not been explored in the ED population through a large multicentred patient cohort.

\section{METHODS}

\section{Study design}

This is a multicentre retrospective cohort study designed to quantify the potential impact of improving ED screening and diagnostic care performance on timely STEMI treatment and post-PCI outcomes. The results are intended to inform the design of a future EHR embedded algorithm to screen for STEMI upon ED arrival. We aim to describe our approach to quantifying the associations between (1) time to diagnosis and (2) time to treatment (PCI) between patients who do and do not receive an early ECG within $10 \mathrm{~min}$. We seek to understand variability in achieving timely PCI, hospital length of stay (LOS), subsequent heart failure and mortality by patient characteristics (ie, age, gender, race, language) and care process factors (ie, achieving timely screening, time of day, distance between ED and cath lab) through these prespecified subgroup analyses. We received institutional review board (IRB) approval from all participating facilities prior to study initiation. A shared IRB approval process was used for this National Institute of Health-funded study.

\section{Study setting}

Participating sites are tertiary care centre EDs within a hospital designated as a PCI centre where the ED physician can activate the cardiac catheterization (cath) lab

\section{Table 1 Definition of time stamps and intervals in STEMI screening and diagnosis}

TIME STAMP CARE INTERVAL DEFINITION

Symptom onset time Time of symptoms prior to arrival

Recalled patient reported time for when symptoms associated with the acute STEMI encounter began.

\section{Time zero}

\begin{tabular}{cll} 
Door time & $\begin{array}{l}\text { ED arrival time } \\
\text { (primary analysis) }\end{array}$ & First recorded presence of the patient in the ED. \\
\hline Screening & $\begin{array}{l}\text { Door to screening } \\
\text { Door-to-first-ECG time, D2E } \\
\text { (primary independent variable of interest) }\end{array}$ & $\begin{array}{l}\text { ED arrival to completion of the first ECG. The first } \\
\text { ECG is generally performed prior to the ED physician } \\
\text { evaluation for the purpose of enabling the early } \\
\text { identification of STEMI. }\end{array}$
\end{tabular}

\section{Diagnosis}

$\begin{array}{lll}\text { Diagnostic ECG time } & \begin{array}{l}\text { Door-to-diagnostic ECG, D2E } \\ \text { (secondary independent Variable of } \\ \text { Interest) }\end{array} & \begin{array}{l}\text { ED arrival to completion of ECG used to activate the } \\ \text { cath lab. }\end{array} \\ \text { Cath lab activation time } & \begin{array}{l}\text { Door-to-catheterisation laboratory } \\ \text { activation }\end{array} & \begin{array}{l}\text { ED arrival to the time when the cath lab was activated } \\ \text { (Code STEMI). }\end{array}\end{array}$

\section{Treatment}

\begin{tabular}{|c|c|c|}
\hline $\begin{array}{l}\text { Patient arrives in cath } \\
\text { lab }\end{array}$ & $\begin{array}{l}\text { Door-to-catheterisation laboratory arrival } \\
\text { time, } \\
\text { diagnostic team centric } \\
\text { (primary outcome) }\end{array}$ & ED arrival to patient arrival in the cath lab. \\
\hline Balloon time & $\begin{array}{l}\text { Door-to-balloon time, } \\
\text { Intervention team centric outcome } \\
\text { (primary outcome) }\end{array}$ & $\begin{array}{l}\text { Time from ED arrival to time the catheterisation } \\
\text { guidewire crossed the culprit coronary lesion in } \\
\text { patients receiving balloon angioplasty. }\end{array}$ \\
\hline
\end{tabular}

Time zero, start time for emergency care. Outcomes, treatment times for patient with STEMI directed to percutaneous coronary intervention. Cath Lab, cardiac catheterisation laboratory; ED, emergency department. 
for emergency STEMI intervention (Code STEMI) with a single phone call. ${ }^{10}$

\section{Process measures}

Exploring STEMI process measures includes quantifying time intervals associated with STEMI screening and diagnosis. Door-to-screening (D2S) defined as the time from ED arrival to the completion of the first ECG (table 1). The time of ECG completion was selected to mark the end of screening because it is the only retrospective clinical timestamp recorded to represent the completion of STEMI screening among those who screen positive. It is typical practice in EDs for ECGs to be taken directly to an emergency physician for interpretation. ${ }^{14-17}$ Door-to-diagnosis (D2D) is the interval from ED arrival to STEMI diagnosis (table 1). STEMI diagnosis is defined as the time when the physician activates a cardiac lab team for emergent PCI. As a result, we primarily measure the completion of diagnosis as door-to-cathlab activation (D2CLA). We found that cath lab activation time was rarely included in the medical record, maintained in an external telephone call centre database and inconsistently recorded. As a result, site principal investigators (PIs) were permitted to export cath lab activation times from their local database for the National Cardiovascular Data Registries' (NCDR) Acute Coronary Treatment and Intervention Outcomes Network (ACTION) Get with the Guidelines Registry supported by the American College of Cardiology (NCDR-ACTION, table 2). We also included the time-to-diagnostic ECG as a secondary measure for diagnostic time.

These definitions are a necessary change from the traditional use of door to ECG as the starting point for STEMI performance measures and reflect how screening and diagnosis require separate metrics for appropriate diagnostic performance evaluation. Delayed STEMI screening and diagnosis are barriers to effective treatment access. By limiting our population to patients screened by the $\mathrm{ED}$, we limit the variation in point-of-first-medical contact to those brought in by emergency medical services (EMS) or self-transport.

\section{Patient and public involvement}

The study research question and outcome measures were developed from a desire to evaluate how well ED STEMI screening and diagnosis are performed for individual patients. We seek to better understand the demographics and presentations of patients who may experience differential outcomes potentially associated with suboptimal STEMI screening. Patients, however, were not directly involved in the design or conduct of this study.

\section{Inclusion/exclusion criteria}

We will include all 2014-2016 ED patients with a final hospital diagnosis of STEMI. To reduce misclassification bias, STEMI will be defined by International Classification of Disease (ICD-9) 9 codes previously validated in the literature and the corresponding ICD-10 diagnosis codes
Table 2 Study data permitted for import from local NCDRACTION Registry databases

\begin{tabular}{ll}
\hline Study variable & $\begin{array}{l}\text { NCDR-ACTION variable } \\
\text { number }\end{array}$ \\
\hline Birth date & 2050 \\
Sex & 2060 \\
Race & 2070 (white) \\
& 2071 (black) \\
& 2073 (American Indian/ \\
& Alaskan Native) \\
& 2072 (Asian) \\
& 2074 (Native Hawaiian/ \\
& Pacific lslander) \\
& 2076 (Hispanic vs non- \\
Ethnicity & Hispanic) \\
& 3300 (private) \\
Health insurance & 3301 (Medicare) \\
& 3302 (Medicaid) \\
& $3303+3304+3305+3306$ \\
(other) \\
ED discharge time & 3307 (uninsured/self pay) \\
\hline Cath lab arrival date & 3159 \\
\hline Cath lab arrival time & 7100 \\
\hline Cath lab activation time & 3222 \\
\hline PCl (yes/no) & 7101 \\
\hline
\end{tabular}

*We did not permit the inclusion of any data that would be used for calculated time intervals, the primary outcome or risk factors/ exposures.

ACTION, Acute Coronary Treatment and Intervention Outcomes Network; ED, emergency department; NCDR, National Cardiovascular Data Registries; PCI, percutaneous coronary intervention.

(table 3). ${ }^{1}$ Data abstractors, familiar with the electronic health record (EHR) of their institution, will review electronic patient charts for study data and to determine if the course of care is consistent with acute STEMI. Care is considered inconsistent with acute STEMI if at least two of the following apply: STEMI is not mentioned in the context of a diagnosis, the discharge summary does not include STEMI as a final diagnosis, there is no cath lab intervention, cath lab findings are not consistent with STEMI anatomy or intervention, and an alternative diagnosis is present for which care is most consistent (including non-STEMI, unstable angina and coronary vasospasm among others). It is recognised that some of these patients' anatomy and physiology may generate ECG findings consistent with an appropriate diagnosis of STEMI from the ED. We opted to exclude these patients because the ultimate goal of STEMI screening from the ED is to identify patients who have STEMI and will benefit from emergent removal of an acute thrombus within a coronary artery. This would be the objective of a precision-oriented approach to screening ED patients on arrival for possible STEMI. We retained patients who received care in the ED but had a diagnostic ECG acquired 
Table 3 STEMI International Classification of Disease Codes (ICD) for Inclusion by final hospital diagnosis

\begin{tabular}{|c|c|c|c|c|c|}
\hline \multicolumn{6}{|c|}{ Acute myocardial infarction (AMI) diagnosis codes associated with STEMI } \\
\hline ICD 9 & Diagnosis & & IC10 & Diagnosis & \\
\hline 410 & AMI & Location & 121 & STEMI and NSTEMI & Location \\
\hline 410.31 & AMI inferoposterior wall & & I21.19 & $\begin{array}{l}\text { STEMI other coronary } \\
\text { artery inferior }\end{array}$ & Inferior \\
\hline \multirow[t]{2}{*}{410.11} & AMI other anterior wall & & 121.02 & STEMI LAD & Anterior \\
\hline & & & 121.09 & $\begin{array}{l}\text { STEMI other coronary } \\
\text { artery anterior }\end{array}$ & Anterior \\
\hline 410.51 & AMI other lateral wall & Lateral & 121.29 & STEMI another sites & Other specified \\
\hline 410.91 & AMI unspecified site & Non-specified & 121.3 & STEMI unspecified & Non-specified \\
\hline
\end{tabular}

*410.81 includes papillary muscle rupture.

LAD, left anterior descending artery; LCX, left circumflex artery; NSTEMI, non-ST-segment elevation myocardial infarction; RCA, right coronary artery; STEMI, ST-segment elevation myocardial infarction.

prior to hospital arrival. Their door-to-diagnostic ECG time would be negative and reflect an opportunity for an alternative care pathway, such as prehospital arrival cath lab activation. Cases inconsistent with acute STEMI are referred to the site PI for chart review. All excluded cases are shared with the Vanderbilt Emergency Care Health Services Research Data Coordinating Centre (HSR-DCC) central study PI (MYABY) for approval. Patients for exclusion are flagged for exclusion by the HSR-DCC but not removed.

\section{Primary outcomes}

The primary outcome (table 1) is door-to-treatment, that is, time from ED arrival to STEMI treatment. An early ECG is defined per existing clinical practice guidelines $^{2314}$ as the time between ED arrival (the patient's first recorded presence in the ED) to the completion of the first ECG in the ED intended to permit the early diagnosis of STEMI. ED arrival or 'door' time is defined as the patient's first recorded presence in the ED. ${ }^{111418}$ Our definition for time to treatment includes two outcome measures. The first measure is door-tocathlab- arrival (D2CAR), a diagnostic team-oriented measure (table 1). Patient cath lab arrival marks the last point in the STEMI care pathway the diagnostic team can influence. The second measure is door-to-balloon (D2B) time, the more traditionally used PCI treatment time measure (figure 1). During the study design phase, we found that D2B time was not consistently documented in the EHR at any of our seven hospitals. Thus, we modified definitions for this timestamp after considering the use of alternative data as established by the NCDR-ACTION Registry. The registry includes proxies for this outcome in a hierarchy such that D2B time can be measured primarily as balloon inflation time, yet the time the guidewire crosses the coronary lesion can be used when D2B time is missing. ${ }^{10} 19$

\section{Secondary outcomes}

Secondary outcomes include ED length of stay (LOS), hospital LOS, change in cardiac ejection fraction (EF) after the acute STEMI and 1-year mortality. ED LOS is defined as the time from ED arrival to ED departure. ${ }^{18}$ Change in EF is calculated as the difference between the last EF measured prior to the patients with STEMI and the first documented after hospital discharge. Hospital LOS is the time from hospital admission to hospital discharge. Mortality at 1 year was assessed by assigning one of three categories to a patient's survival status 1 year after the STEMI ED visit: deceased (with date, time and cause noted), alive (based on evidence of contact with the health system via EHR documentation) and lost to follow-up.

\section{Risk factors}

The independent variable of primary interest is time to screening defined as door-to-first ECG (D2E $\mathrm{D}_{1 \mathrm{st}}$ ) (table 1). This is the screening (D2S) time interval measured as both a continuous variable and dichotomised (D2S $\leq 10 \mathrm{~min}$ vs $\mathrm{D} 2 \mathrm{~S}>10 \mathrm{~min}$ ) per existing clinical practice guidelines. ${ }^{23}$ Additional risk factors of interest include information often known about a patient on ED arrival which will be examined in exploratory analyses as adjusting variables. These include age, gender, race, primary language, arrival time (time of day), arrival mode (EMS, self-transport or other) and chief complaint. 


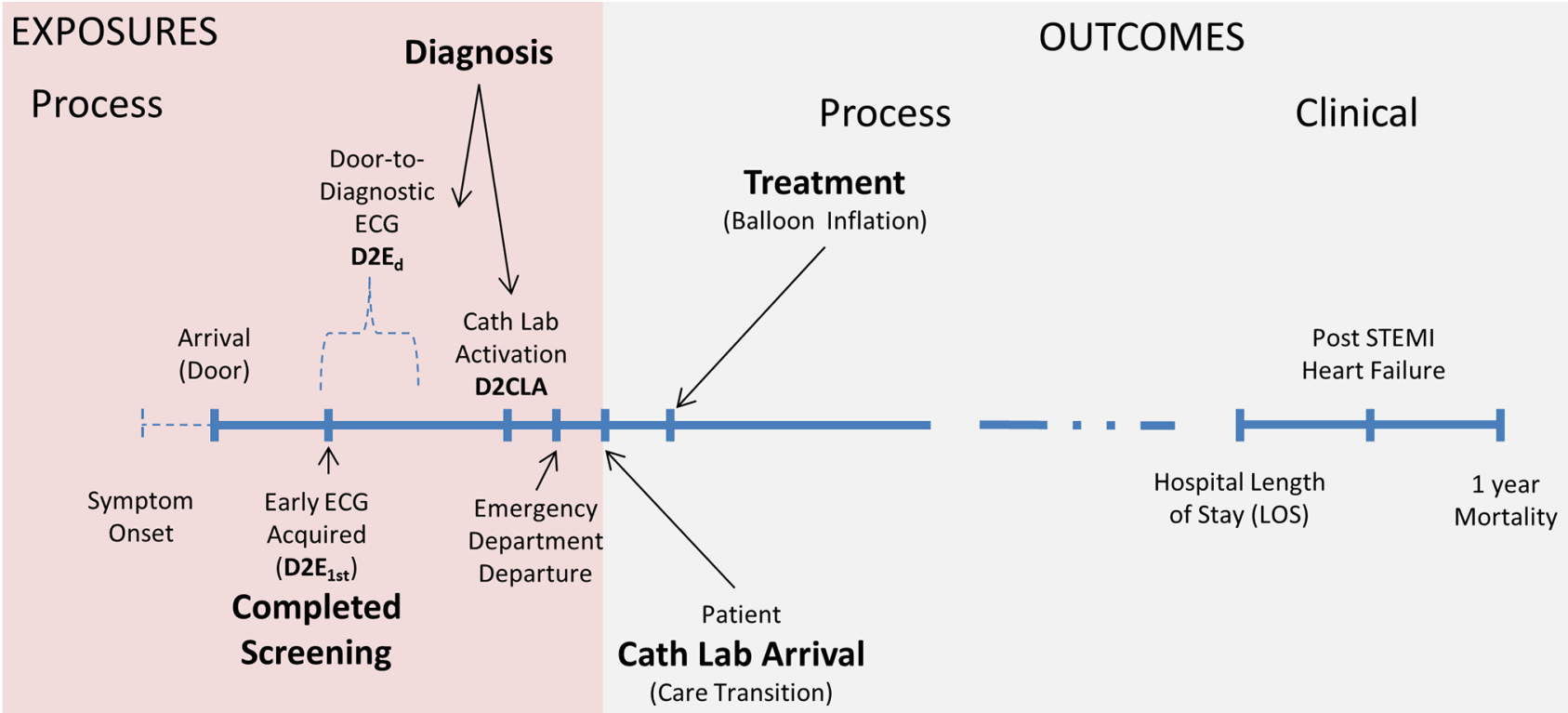

\begin{tabular}{|c|c|c|c|}
\hline SCREENING & DIAGNOSIS & TREATMENT & CLINICAL OUTCOME \\
\hline Door-to-Screening & \multicolumn{2}{|l|}{ D2S } & \multirow{2}{*}{$\begin{array}{l}\text { Clinically Meaningful } \\
\text { STEMI Process Measures }\end{array}$} \\
\hline Door-to-Diagnosis & D2L & \multirow[b]{2}{*}{ D2CAR } & \\
\hline \multicolumn{2}{|c|}{ Door-to-Cathlab-Arrival } & & Across the Care Continuum \\
\hline Door-to-Balloon & & D2B & \\
\hline
\end{tabular}

Figure 1 STEMI patient care process measures: Screening, Diagnosis and Treatment $\mathrm{D}_{2} \mathrm{E}_{1 \mathrm{st}}=$ door-to-first ECG=door-to-early $E C G=$ doorto screening (D2S). D2E=door-to-diagnostic ECG=one of two ways to measure door to diagnosis (D2D). More ideally, D2D can be measured as door-to-catheterisation laboratory activation (D2CLA). Prehospital ECGs interpreted by the paramedic team as a STEMI would be represented as 'negative' door-to-diagnostic ECG time. These patients would ideally bypass the ED care pathway in the absence of an over-riding need for non- $\mathrm{PCl}$ (or pre- $\mathrm{PCl}$ ) care (ie, motor vehicle collision injuries requiring stabilisation, witnessed cardiac arrest after prehospital ECG acquisition, etc). Thus, negative D2S would indicate potential opportunity for an alternative care pathway.

\section{Secondary subgroup analysis}

We also included patient characteristics known to increase the risk for STEMI and to be associated with outcome differences. ${ }^{314}$ These include symptom onset, ${ }^{19-21}$ as well as a history of diabetes (prediabetes was not included), hypertension, dyslipidaemia, tobacco use, heart failure, prior myocardial infarction, prior coronary artery bypass graft and prior PCI procedure. In defining variables, we balanced maximising covariate granularity with medical informatics best practice for data integration and data standardisation. For example, tobacco use status is recorded by NCDR as a dichotomous variable. In order to obtain more details, we collected these data primarily from the EHR. During the study design phase, we evaluated the smoking history data available in each EHR and found the degree of tobacco exposure was variably categorised across our seven sites. We developed the following categories to maximise variability while standardising data reporting: current smoker, prior smoker but quit and non-smoker. Tobacco exposure fields in the shared database were limited to only accept one of these three smoking status designations for each patient. ${ }^{22} 23$
Recognising the impact of EHR user access and data use context, ${ }^{21}$ we only include information available to the diagnostic care providers at the time of the initial encounter. These providers are typically the ED team but can include an interventional cardiology consultant for rare presentations or complex patients. The NCDR-ACTION Registry permits the inclusion of all data available on review of the full medical record. The structure of the ED interface with EHRs varies between hospitals with some having more or less data available on patient arrival. As a result, we opted for data collection directly from the EHR using what is accessible during the early phases of the diagnostic clinical encounter.

\section{Sample size}

We estimate our analysis will require 1220 patients from our seven study sites. This was based on our plan for a non-parametric Wilcoxon rank-sum test comparing D2B time between dichotomised door-to-first-ECG (D2E) groups of patients with STEMI: early ECG (D2E $\leq 10$ minutes) vs. missed screening (D2E $>10$ minutes). An aggregation of ICD 9/10 code counts within each hospital 
from a prior studies suggests approximately 444 ED patients with STEMI are seen in these seven EDs annually with $87.2 \%$ captured with a timely early ECG and $12.8 \%$ in the missed screening cases. ${ }^{14}{ }^{24}$ This is the effective sample size required to detect a standardised difference of 0.35 , with a type I error rate of 0.05 and power of $80 \%$ in two-tailed tests. This is a small to medium effect size by Cohen's nomenclature. ${ }^{25}$ This translates to 596 patients and a detectable D2B time difference of 5.2 min. ${ }^{26}$ Due to potential correlation in D2B between patients seen at the same ED, we calculated a cluster design effect of 1.84 assuming an intercluster correlation coefficient of 0.01 . This required us to include a minimum of 1220 patients. With an anticipated ICD coding misclassification exclusion rate of $5 \%-10 \%$, this patient sample size is achievable with 3 years of data.

\section{Data collection}

Cohort data for patients meeting study year, ED care and ICD diagnosis code inclusion criteria are extracted from each hospital's EHR using a preprogrammed report to identify the study cohort. These data are sent securely to the HSR-DCC using the HIPPA and research data security 'Sendit' function of Research Electronic Data Capitulation (REDCap). REDCap is a secure, web-based application designed exclusively to support data capture for research studies. ${ }^{27-29}$ The cohort data for each site are uploaded into a subsection of the larger study database built and maintained by the HSR-DCC. The coordinating study PI and HSR-DCG staff have access to all study data, but individual sites only see their patient records. The use of a centrally designed database with built-in variable definitions and quality control checks ensured data harmonisation across sites. ${ }^{27} 30$

At a minimum, cohort data include a patient identifier (typically the medical record number), ED date of service and final hospital ICD diagnosis codes. Each patient record is reviewed by a data abstractor associated with each institution's ED. A REDCap-based data collection form is completed with existing EHR data that, as noted above, reflect information available to diagnostic providers in the ED during the clinical encounter. Prior to data collection, each site PI completed a training case form (TCF, box 1) in which data were collected for the first patient of record for study inclusion. The location of each variable within the EHR, including the location within specific documents, was recorded and used as a guide for the local data abstractors. The resultant data dictionary was used to verify data definitions were standardised across sites. In total, we had 11 data abstractors from the 7 EDs. All data abstractors received a minimum of 2 hours of training to further ensure standardised data collection. Training was via a two-part module developed and delivered by the HSR-DCC. Part 1 involved a $90 \mathrm{~min}$ session via video conference introducing the study design, the data abstractors' role in the project, study data definition and practice using all fields of the study database for the TCF patient. Part 2 involved repeating the data entry

\section{Box 1 Data abstractor training module}

Part 1: 90 min video conference

- Content

- Clinical problem: What is known and unknown about STEMI and STEMI patient outcomes

Study questions

- Study design

Clinical care pathway for STEMI care:

Case study: Beverly Hospital, Beverly, Massachusetts

- https://www.youtube.com/watch?v=FkeHO36oigo (View to $3 \mathrm{~min}$ and $50 \mathrm{~s}$ )

- Role of the ECG

PCl procedure:

Clinical timestamps and care documentation in the EHR

- The procedure: $\mathrm{PCl}$ care and timestamps:

- https://www.youtube.com/watch?v=I45kJJoCa6s: (View full video)

- https://www.youtube.com/watch?v=-BuazAhs7uA: (View to $1 \mathrm{~min}$ and $10 \mathrm{~s})$.

- Outcome measure data definitions (similarities and differences with NCDR-Get with the Guidelines ACTION Registry

- Study procedures and timeline

Introduction to study database

Data entry with Training Case Form (TCF) example

- Part 2: Independent data abstraction for TCF patient directly form the local EHR (30 min)

- A Data Abstractor is approved to start data entry after Emergency Care Health Services Research Data Coordinating Center staff review and confirm accurate and complete TCF data entry within the database for the TCF patient. Once confirmed, the site PI co-signs a delegation of authority form certifying the Data Abstractor is trained and will collect data under their guidance.

ACTION, Acute Coronary Treatment and Intervention Outcomes Network; EHR, electronic health record; NCDR, National Cardiovascular Data Registries; PCl, percutaneous coronary intervention; PI, principal investigator; STEMI, STsegment elevation myocardial infarction.

process for the TCF patient with direct use of the associated EHR record (figure 2).

We verified that all participating ED sites submit data for patients with STEMI to the NCDR-ACTION Registry. Despite the presence of this existing data registry, we undertook primary data collection for additional information on ED-level STEMI care variables. Site PIs, however, were permitted to send select variables with identical data definitions to HSR-DCC data from their local NCDR-ACTION Registry database (table 2). These data were uploaded directly into the database by the HSR-DCC to reduced data entry time, and verified by data abstractors on chart review.

The data collection form within the study database has alerts for values outside of the expected range and instructions for uniform units of measure. The HSR-DCC staff review all completed entries for accuracy with the use of data cleaning checks run via R-statistical code (www. r-project.org, available at http://biostat.mc.vanderbilt. edu/wiki/Main/JenkinsEMCode) on data contained in the study database after the completion of 2014 data, 2015 data and study close. The data cleaning code identifies 
Variable Name

PATIENT DEMOGRAPHICS

Primary Language

EMERGENCY DEPARTMENT - ED

Triage start time

Triage end time

Emergency Severity Index (ESI) score

Measure of anticipated care acuity assigned upon ED Triage

(lower $=$ higher acuity)

Onset of symptoms prior to arrival in your ED

Measured as hours prior to presentation, no assumptions made for

patient reports of this morning, last night, yesterday, etc

Chief Complaint Reported Upon Arrival

Chest Pain (yes/no)

Chief Complaint Reported Upon Arrival

Chief Complaint 1-5

Final ED Diagnosis

Diagnostic Care Team's Diagnosis 1-5

ED discharge date

\section{HOSPITALISATION}

Hospital Discharge Diagnosis ICD codes 1-5

Action includes the first 3 rather than 5

Was there a First PCI Center ED ECG?

PCI Center Early ECG

- First PCI Center ED ECG date

- First PCI Center ED ECG time

- First PCI Center ED ECG Clinical Interpretation

- First PCI Center ED ECG Official ECG Interpretation

\begin{tabular}{|c|c|}
\hline \multicolumn{2}{|c|}{ Receiving Hospital Follow Up ECG } \\
\hline $\begin{array}{l}\text { Was there a follow up ECG at the Receiving hospital? } \\
\text { - Receiving Hospital Follow Up ECG date } \\
\text { - Receiving Hospital Follow Up ECG time } \\
\text { - Official ECG Interpretation } \\
\text { - Clinical Interpretation of Receiving ED EKG }\end{array}$ & Yes/No \\
\hline \multicolumn{2}{|r|}{ Prior ECG } \\
\hline $\begin{array}{l}\text { Was there a prior ECG from an outside facility or agency? If } \\
\text { yes, } \\
\text { - ECG from EMS Transferring to Receiving Hospital } \\
\text { - ECG from Outside Hospital } \\
\text { - ECG by EMS transporting to Outside Hospital } \\
\text { - Referring Clinic Provider ECG }\end{array}$ & $\begin{array}{l}\text { Yes/No } \\
\text { If yes, date and time, clinical interpretation, official interpretation }\end{array}$ \\
\hline \multicolumn{2}{|c|}{ Diagnositic ECG (select one) } \\
\hline $\begin{array}{l}\text { - PCI Center Early ECG } \\
\text { - EMS Transferring from the Field to the PCI Center ED } \\
\text { - Outside Hospital ED } \\
\text { - EMS Transporting from OSH ED } \\
\text { - Referring Clinic Provider }\end{array}$ & ECG with which the decision was made to activate the cath lab emergently \\
\hline
\end{tabular}

Change in Ejection Fraction (Pre, During Index Visit, Post Index Visit)

\begin{tabular}{|l|l|}
\hline \multirow{2}{*}{ Last EF Prior to Index Visit } & $\begin{array}{l}\text { EF measured before this current index visit? (Yes/No) } \\
\text { If yes, } \\
\text { - Prior EF Date } \\
\text { - Prior EF \% (lowest documented) } \\
\text { - Prior EF Range }\end{array}$ \\
\hline EF during Index Visit & $\begin{array}{l}\text { EF measured during index visit? (Yes/No) } \\
\text { If yes, } \\
\text { - Index Visit EF Date } \\
\text { - Index Visit EF \% (lowest document) } \\
\text { - Index Visit EF Range }\end{array}$ \\
\hline EF after Index Visit & $\begin{array}{l}\text { First post index visit discharge EF (Yes/No) } \\
\text { - Post-Index-Visit EF Date } \\
\text { - Post-Index-Visit EF \% (lowest documented) } \\
\text { - Post-Index-Visit EF Range }\end{array}$ \\
\hline
\end{tabular}

Figure 2 Study variables not available in the NCDR-ACTION Registry. ACTION, Acute Coronary Treatment and Intervention Outcomes Network; ED, emergency department; EF, ejection fraction; ICD, International Classification of Disease; NCDR, National Cardiovascular Data Registries; $\mathrm{PCl}$, percutaneous coronary intervention. 
missing values, patterns of missingness and inconsistent data entries (eg, an ED arrival date that occurs before date of birth is likely a data entry error in the year for the ED visit or birth). Results of the first data cleaning checks are communicated to the site PI and data abstractors at the end of 2014 data collection, discussed via telephone conference call, with a response verified by the HSR-DCC staff. Subsequent data checks are run upon request and at a minimum of every 30 days. Results for follow-up data checks are run for each site, then communicated to each collaborating team via email. The full report is then saved on a shared secure drive (vanderbilt.box.com) managed by the HSR-DCC with specific subfolders for each site. Access permissions are set such that data for each site are only seen by the site PI and local data abstractors. Site PIs are asked to clarify ambiguous entries. The HSR-DCC study coordinator follows up on all requests for data clarification.

\section{Data analysis}

Descriptive statistics for screening, diagnosis and treatment time intervals including D2S (door-to-screening), D2D (door-to-diagnosis), D2CLA (door-to-diagnosis communication via cath lab activation), D2CAR (doorto-ED-to-cardiology care transition), D2B (door-to-treatment) and patient characteristics, will be calculated using mean, SDs and quartiles for continuous variables and proportions for categorical variables. They will be compared between the two primary exposure patient with STEMI groups: early ECG and missed screening cases using non-parametric Wilcoxon rank-sum test for continuous variables and $\chi^{2}$ test for categorical variables.

For the primary adjusted analysis, we will first use a linear mixed-effects regression model with $\mathrm{D} 2 \mathrm{~B}$, as the outcome. The primary independent variable of interest is D2S status ( $\mathrm{D}_{2} \mathrm{E}_{1 \mathrm{st}} \leq 10 \mathrm{~min}$ vs $\mathrm{D}_{2} \mathrm{E}_{1 \mathrm{st}}>10 \mathrm{~min}$ ) with a random effect for the ED providing care. We will adjust for ED screening methods (eg, point of first patient contact in the ED, dedicated space for early ECGs, etc), care process factors (eg, time of day, distance between ED and cath lab, etc) and individual patient characteristics. Since D2S is a portion of D2B, we will use the first-ECGto-balloon time interval ${ }^{26}$ calculated by subtracting D2S from D2B as the primary outcome in this model. Results from those adjusted analyses will help quantify differences in timely care between early ECG and missed screening case patients with STEMI and reduction in door-to-treatment (D2B) for every minute saving in time to screening (D2E). These analyses will be repeated with D2CAR as the outcome, then D2D as the independent variable of interest. ${ }^{31}$

We will then perform a time-to-event analysis using the Cox proportional hazard model stratified by ED for each secondary outcome event (hospital LOS and 1-year mortality), and a linear mixed-effects regression model with ED random effect for continuous outcomes (change in cardiac EF after acute STEMI) with the same adjustments and independent variables as the primary analysis.
Lastly, we will use our adjusted data to construct a summary of the care course (the sequence of median STEMI process intervals) by age, gender, race, language, presenting symptom and ED subgroups to identify differences in the following time intervals: symptom-onset-to-arrival, arrival-to-first ECG, first-ECG-to-diagnostic-ECG, diagnostic-ECG-to-cathlab-activation, activation-to-PCI balloon, PCI-to-hospital discharge (see figure 1).

\section{DISCUSSION}

Despite the limitations of retrospective EHR data, we selected this approach over a prospective study for several reasons. First, the time and financial cost of the prospective approach would make the study impracticable. Prospective enrolment would require 4 years to complete data collection and continued screening of ED patients. The cost would outweigh the enrolment yield given the relative infrequency of STEMI events within the larger ED patient population. These logistics would significantly slow our ability to generate knowledge to inform an important study question for a deadly disease. Second, our targeted screening intervention will use EHR data available to the ED care team on arrival, therefore, the use of existing EHR data will be subject to similar data conditions during intervention implementation.

Substantial resources are allocated to assure screening and diagnosis within 10 minutes of patient arrival to achieve timely STEMI treatment. Yet no existing measures or databases have adequate granularity to measure screening and diagnostic practice or to guide performance optimisation. Much of the resource investment reflects the major consequences and medicolegal gravity of a missed STEMI in the context of time limited interventions, high mortality and significant morbidity. If interventions are to be developed to more precisely identify patients with STEMI on ED arrival, data on ED patients with STEMI are critical. These interventions need to be balanced with appropriate use of resources for this infrequent but potentially deadly condition.

Current practices are often supported by data extrapolated from the more broad population of hospital STEMI patients who may be different from the ED subpopulation. This study will increase our understanding of whether those missed by ED STEMI screening receive less timely interventional care (PCI) than those with timely STEMI screening and diagnosis. It will better characterise the care process, demographic profile and clinical outcomes for this subpopulation of patients with STEMI. The primary results of this study will be a comparison of differences in the timeliness of treatment between those who experienced timely versus delayed screening and diagnosis. Our subgroup analysis may identify risk factors for poor outcomes providing data to focus clinical interventions to deliver precise diagnostic care normalised for subgroup-specific risk factors. 
The American Heart Association recently called for growth in the use of linked registry and EHR data to understand the penetration of cardiovascular care guidelines and evidence within clinical practice. ${ }^{32}$ Our methods present an applied approach to the use of EHR data for emergency care sensitive cardiovascular disease diagnoses. NCDR-ACTION Registry is a robust risk-adjusted, outcomes-based, quality improvement programme that focuses exclusively on patients with high-risk STEMI and non-NSTEMI. The registry database has revolutionised our ability to study outcomes for these high-risk conditions despite their relatively low prevalence at any given centre. However, the NCDR-ACTION Registry is focused on treatment performance, and it lacks variables (figure 2) to support evidencebased screening and diagnostic performance evaluation to improve clinical practice. In contemporary practice the existence of EHRs is more the norm than the exception. ${ }^{33}$ EHRs provide a vehicle for source data and the potential application of dynamic clinical decision support to enhance risk stratification and mechanisms for evidence-based care delivery. In this study, we used standardised multicentre primary data collection from seven hospital EHRs to enable our ability to study these early STEMI care performance targets.

The completion of this study will provide a more accurate appraisal and critical feedback on the quality of contemporary STEMI care pathway performance that can be used to improve emergency care delivery for ED patients with STEMI, and inform the development of screening and diagnostic support tools that can be translated to other care environments. Specifically, we will better understand the consequences of and risk factors for delayed screening and diagnosis. We anticipate our results will be extrapolated to other care delivery spaces that receive undifferentiated patients (non-PCI centre EDs and urgent care). What is learnt about differential risk may be applied in primary care clinics, intake processes for direct to floor admissions and interservice floor transfers. Tools developed to improve screening may be used for other emergency care sensitive conditions.

\section{ETHICS DISSEMINATION}

Manuscript publication is our primary plan for results dissemination. Given the critical nature of STEMI, we plan to simultaneously share our study results with the participating institutions STEMI care quality improvement committees, Divisions of Cardiology as well as ED leadership. The study data will be available to other researchers on a case-by-case basis via the Vanderbilt University Emergency Care Health Services Research Data Coordinating Center (HSR-DCC). Statistical code will be made available on the HSR-DCC website.

\section{Author affiliations}

${ }^{1}$ Department of Emergency Medicine, Vanderbilt University, Nashville, Tennessee, USA
${ }^{2}$ Department of Emergency Medicine, University of California at Davis, Sacramento, California, USA

${ }^{3}$ Department of Emergency Medicine, Brigham and Women's Hospital, Harvard University, Boston, Massachusetts, USA

${ }^{4}$ Department of Emergency Medicine, University of Wisconsin Madison, Madison, Wisconsin, USA

${ }^{5}$ Department of Emergency Medicine, Columbia University Medical Center, New York, USA

${ }^{6}$ Department of Emergency Medicine, Parkland Hospital, University of Texas

Southwestern, Dallas, Texas, USA

${ }^{7}$ Department of Emergency Medicine, Oregon Health and Sciences University, Portland, Oregon, USA

${ }^{8}$ Department of Biostatistics, Vanderbilt University, Nashville, Tennessee, USA

${ }^{9}$ Owen Graduate School of Management, Vanderbilt University, Nashville, Tennessee, USA

${ }^{10}$ Department of Biomedical Informatics, Vanderbilt University, Nashville, Tennessee, USA

${ }^{11}$ Division of Cardiology, Department of Medicine, Vanderbilt University, Nashville, Tennessee, USA

${ }^{12}$ Department of Medicine, Vanderbilt University, Nashville, Tennessee, USA

${ }^{13}$ Division of Critical Care, Department of Medicine, Vanderbilt University, Nashville, Tennessee, USA

Acknowledgements Special thanks to the data abstractors at the seven study sites including: Caitlin Azzo, Oluyemi 0 Olubowale, Alex Trinh, Shannon McNabb, Samita Kumar, Sean Harla, Dr Margo Kaller, Dr Jane Dyball, Dr Daniel Steward and Dr Christopher Beck. We are appreciative of Christina Kampe (Vanderbilt Emergency Care Health Services Research Data Coordinating Center's IRB Regulatory and Compliance Specialist) for managing the multisite IRB approval process that was critical to initiate this study.

Contributors MYABY designed and coordinated the logistics of the study and served as lead investigator via the data coordinating centre. BEM, CWB, BWP, AMM, GS and MT are site principal investigators and coordinate study implementation within their institutions. CAJ is the lead staff biostatistician. KFM is the data coordinating centre's Data Integrity Officer. BEJ is the study programme manager. JLW and SCD were site data abstractors who contributed to study methods development. CUL, TJW, SPC, ABS and RSD provided content-specific expertise advising study design and implementation. TJV and GRB provided content-specific data analysis guidance. DL was the overseeing statistician who codesigned the study with MYABY and will oversee final data analysis with MYABY.

Funding Research reported in this publication was supported by the National Heart Lung and Blood Institute's (NHLBI) award numbers 5K12HL109019, 1K23HL133477, 5K08HL130546.

Disclaimer The content is solely the responsibility of the authors and does not necessarily represent the official views of the National Institutes of Health.

Competing interests MYABY is Director of the Emergency Department Operations Study Group (EDOSG). CWB is a member of the Advisory Board, consultant for Roche Diagnostics and Janssen Pharmaceuticals, and has received research funding from Boehringer Ingelheim. ABS has also received grant support from Abbott Diagnostics and Roche Diagnostics. He is a consultant for Roche Diagnostics, Novartis Pharmaceuticals Corp, Alere Diagnostics, Trevena, Beckman Coulter and Siemens. SPC received grant research support from NIH/NHLBI, PCORI, Cardiorentis, Novartis and Cardioxyl and consultant support/other from Novartis, Trevena, Cardiorentis, Cardioxyl and Siemens.

Patient consent Obtained.

Ethics approval Ethics approval was obtained from the Vanderbilt IRB, IRBChoice (a single IRB approval mechanism used by University of Pennsylvania and Oregon Health and Sciences University), and the institutional IRBs of Brigham and Women's Hospital, University of California at Davis, and University of Wisconsin.

Provenance and peer review Not commissioned; peer reviewed for ethical and funding approval prior to submission.

Open Access This is an Open Access article distributed in accordance with the Creative Commons Attribution Non Commercial (CC BY-NC 4.0) license, which permits others to distribute, remix, adapt, build upon this work non-commercially, and license their derivative works on different terms, provided the original work is properly cited and the use is non-commercial. See: http://creativecommons.org/ licenses/by-nc/4.0/ 
(c) Article author(s) (or their employer(s) unless otherwise stated in the text of the article) 2018. All rights reserved. No commercial use is permitted unless otherwise expressly granted.

\section{REFERENCES}

1. Ward MJ, Kripalani S, Zhu Y, et al. Incidence of emergency department visits for ST-elevation myocardial infarction in a recent six-year period in the United States. Am J Cardiol 2015;115:167-70.

2. Steg PG, James SK, Atar D, et al. ESC Guidelines for the management of acute myocardial infarction in patients presenting with ST-segment elevation. Eur Heart J 2012;33:2569-619.

3. O'Gara PT, Kushner FG, Ascheim DD, et al. 2013 ACCF/AHA Guideline for the management of st-elevation myocardial infarction. $J$ Am Coll Cardiol 2013;61:e78-140.

4. Wessler JD, Stant J, Duru S, et al. Updates to the ACCF/AHA and ESC STEMI and NSTEMI guidelines: putting guidelines into clinical practice. Am J Cardiol 2015;115-23A-8.

5. Carrillo X, Fernandez-Nofrerias E, Rodriguez-Leor O, et al. Early ST elevation myocardial infarction in non-capable percutaneous coronary intervention centres: in situ fibrinolysis vs percutaneous coronary intervention transfer. Eur Heart J 2016;37:1034-40.

6. McNamara RL, Wang Y, Herrin J, et al. Effect of door-to-balloon time on mortality in patients with ST-segment elevation myocardial infarction. J Am Coll Cardiol 2006;47:2180-6.

7. Gibson CM, Pride YB, Frederick PD, et al. Trends in reperfusion strategies, door-to-needle and door-to-balloon times, and in-hospital mortality among patients with ST-segment elevation myocardia infarction enrolled in the National Registry of Myocardial Infarction from 1990 to 2006. Am Heart J 2008;156:1035-44.

8. Mehta $\mathrm{RH}$, Bufalino VJ, Pan W, et al. Achieving rapid reperfusion with primary percutaneous coronary intervention remains a challenge: insights from American Heart Association's get with the guidelines program. Am Heart J 2008;155:1059-67.

9. French WJ. Trends in acute myocardial infarction management: use of the National Registry ofMyocardial Infarction in quality improvement. Am J Cardiol 2000;85:5-9.

10. Henry TD, Sharkey SW, Burke MN, et al. A regional system to provide timely access to percutaneous coronary intervention for ST-elevation myocardial infarction. Circulation 2007;116:721-8.

11. Diercks DB. Triage of emergency department patients with chest pain: where should we set the bar? Ann Emerg Med 2009;53:746-7.

12. Graff L, Palmer AC, Lamonica $P$, et al. Triage of patients for a rapid (5-minute) electrocardiogram: A rule based on presenting chief complaints. Ann Emerg Med 2000;36:554-60.

13. Sinnaeve PR, Van de Werf F. Transporting STEMI patients for primary PCl: a long and winding road paved with good intentions? Eur Heart J 2016;37:1041-3.

14. Yiadom MY, Baugh CW, McWade CM, et al. Performance of emergency department screening criteria for an early ecg to identify ST-Segment elevation myocardial infarction. J Am Heart Assoc 2017;6:e003528.

15. Yiadom M, Liu X, McWade CM, et al. Acute coronary syndrome screening and diagnostic practice variation. Acad Emerg Med 2017:24:701-9

16. Diercks DB, Kirk JD, Lindsell CJ, et al. Door-to-ECG time in patients with chest pain presenting to the ED. Am J Emerg Med 2006;24:1-7.
17. Menees DS, Peterson ED, Wang Y, et al. Door-to-balloon time and mortality among patients undergoing primary $\mathrm{PCl}$. N Engl J Med 2013;369:901-9.

18. Yiadom MY, Scheulen J, McWade CM, et al. Implementing data definition consistency for Emergency Department Operations Benchmarking and Research. Acad Emerg Med 2016;23:796-802.

19. Messenger JC, Ho KKL, Young CH, et al. The National Cardiovascular Data Registry (NCDR) Data Quality Brief. J Am Coll Cardiol 2012;60:1484-8.

20. Peterson ED, Roe MT, Chen AY, et al. The NCDR ACTION RegistryGWTG: transforming contemporary acute myocardial infarction clinical care. Heart 2010;96:1798-802.

21. Form DC. Acute coronary treatment and intervention outcomes network registry: National Cardiovascular Data Registry. 2007. https://www.ncdr.com/WebNCDR/docs/default-source/public-datacollection-documents/action_v2_datacollectionform_2-4_limited. pdf?sfvrsn=2

22. Bakken S. An informatics infrastructure is essential for evidencebased practice. J Am Med Inform Assoc 2001;8:199-201.

23. Botsis T, Hartvigsen G, Chen F, et al. Secondary use of EHR: data quality issues and informatics opportunities. AMIA Jt Summits Trans Sci Proc 2010;2010:1-5.

24. Masoudi FA, Magid DJ, Vinson DR, et al. Implications of the failure to identify high-risk electrocardiogram findings for the quality of care of patients with acute myocardial infarction: results of the Emergency Department Quality in Myocardial Infarction (EDQMI) study. Circulation 2006;114:1565-71.

25. Cohen J. A power primer. Psychol Bull 1992;112:155-9.

26. Bradley EH, Herrin J, Wang Y, et al. Door-to-drug and door-toballoon times: where can we improve? Time to reperfusion therapy in patients with ST-segment elevation myocardial infarction (STEMI). Am Heart J 2006;151:1281-7.

27. Harris PA, Taylor R, Thielke R, et al. Research electronic data capture (REDCap)--a metadata-driven methodology and workflow process for providing translational research informatics support. J Biomed Inform 2009;42:377-81.

28. Obeid JS, McGraw CA, Minor BL, et al. Procurement of shared data instruments for Research Electronic Data Capture (REDCap). J Biomed Inform 2013;46:259-65.

29. Harris PA. Research Electronic Data Capture (REDCap) - planning, collecting and managing data for clinical and translational research. BMC Bioinformatics 2012;13:A15.

30. Bloomrosen M, Detmer DE, Informatics DDE. Informatics, evidencebased care, and research; implications for national policy: a report of an American Medical Informatics Association health policy conference. J Am Med Inform Assoc 2010;17:115-23.

31. Niles NW, Conley SM, Yang RC, et al. Primary percutaneous coronary intervention for patients presenting with ST-segment elevation myocardial infarction: process improvement in a rural ST-segment elevation myocardial infarction receiving center. Prog Cardiovasc Dis 2010;53:202-9.

32. Maddox TM, Albert NM, Borden WB, et al. The learning healthcare system and cardiovascular care: a scientific statement from the American Heart Association. Circulation 2017;135:e826-57.

33. Charles D, Gabriel M, Furukawa MF. Adoption of electronic health record systems among US non-federal acute care hospitals: 2008-2014. ONC data brief 2015;9:1-10. 